\title{
Equational characterization for two-valued states in orthomodular quantum systems
}

\author{
G. Domenech* ${ }^{* 1,4}$, H. Freytes* ${ }^{2,3}$ and C. De Ronde ${ }^{4,5}$ \\ 1. Instituto de Astronomía y Física del Espacio (IAFE) \\ Casilla de Correo 67, Sucursal 28, 1428 Buenos Aires - Argentina \\ 2. Universita degli Studi di Cagliari, Via Is Mirrionis 1, 09123, Cagliari - Italia \\ 3. Instituto Argentino de Matemática (IAM) \\ Saavedra 15 - 3er piso - 1083 Buenos Aires, Argentina \\ 4. Center Leo Apostel (CLEA) \\ 5. Foundations of the Exact Sciences (FUND) \\ Brussels Free University Krijgskundestraat 33, 1160 Brussels - Belgium
}

\begin{abstract}
In this paper we develop an algebraic framework in which several classes of two-valued states over orthomodular lattices may be equationally characterized. The class of two-valued states and the subclass of Jauch-Piron two-valued states are among the classes which we study.
\end{abstract}

Keywords: two-valued states, orthomodular lattices, varieties PACS numbers: 02.10 De

\section{Introduction}

In the tradition of the quantum logical research, a property of (or a proposition about) a quantum system is related to a closed subspace of the Hilbert space $\mathcal{H}$ of its (pure) states or, analogously, to the projector operator onto that subspace. Each projector is associated to a dichotomic question about the actuality of the property [23, pg. 247]. A physical magnitude $M$ is represented by an operator $\mathbf{M}$ acting over the state space. For bounded

${ }^{*}$ Fellow of the Consejo Nacional de Investigaciones Científicas y Técnicas (CONICET) 
self-adjoint operators, conditions for the existence of the spectral decomposition $\mathbf{M}=\sum_{i} a_{i} \mathbf{P}_{i}$ are satisfied. The real numbers $a_{i}$ are interpreted as the outcomes of the measurements of the magnitude $M$ and projectors $\mathbf{P}_{i}$ as events. The physical properties of the system or events are organized in the orthomodular lattice of closed subspaces $\mathcal{L}(\mathcal{H})=\langle\mathcal{P}(\mathcal{H}), \vee \wedge, \neg, \mathbf{0}, \mathbf{1}>$. This first event structure was introduced in the thirties by Birkhoff and von Neumann [2]. In this frame, the pure state of the system may be represented by the meet (i.e. the lattice infimum) of all actual properties or, equivalently, as a measure $s: \mathcal{P}(\mathcal{H}) \rightarrow[0,1]$ satisfying

$$
s(0)=0 ; s(\neg \mathbf{P})=1-s(\mathbf{P}) ; s\left(\bigvee \mathbf{P}_{i}\right)=\sum s\left(\mathbf{P}_{i}\right)
$$

with $\left\{\mathbf{P}_{i}\right\}$ a denumerable orthogonal family and $\neg \mathbf{P}$ standing for the orthogonal complement of $\mathbf{P}$.

Different kinds of states have been deeply investigated within the quantum logical program not only because of their importance in order to understand quantum mechanics $[11,12,25,28]$, but also because they provide different representations of the event structure of quantum systems [21, 32, 33].

Recently, several authors have paid attention to the study of states over extended algebraic structures, directly or indirectly related to quantum mechanics, as orthomodular posets [5, 26], $M V$-algebras [7, 15, 16, 22, 27] or effect algebras $[9,29,30]$. Common open problems of these structures are the characterization of classes of algebras admitting some special types of states $[10,20]$ and the internalization in an algebraic structure of the concept of state $[6,17]$.

The aim of this paper is to investigate and equationally characterize classes of two-valued states acting over orthomodular lattices. To do this, we enlarge the language of the orthomodular lattices with a unary operator $s$, satisfying a set of equations, that captures the common properties of several classes of two-valued states. The resulting class is a variety of lattices called orthomodular lattices with internal Boolean pre-state or $I E_{B}$-lattices for short.

The paper is structured as follows. In Section 1 we recall some basic notions of universal algebra and orthomodular lattices. In Section 2 we briefly review the importance of two-valued states in relation to the hidden variables program and representation theorems for orthostructures. In Section 3, we introduce the notion of Boolean pre-state and study its properties. Orthomodular lattices with an internal Boolean pre-state $\left(I E_{B^{-}}\right.$ lattices) are defined and characterized. In Section 4 we relate the category of 
$I E_{B}$-lattices with the category of orthomodular lattices that admits Boolean pre-states through a functor. In Section 5 we provide a categorical equivalence between arbitrary subcategories of orthomodular lattices admitting Boolean pre-states and classes of directly indecomposable $I E_{B}$-lattices. The next two sections are devoted to apply this categorical equivalence to obtain equational systems that characterize the class of two valued states and the subclass of Jauch-Piron two-valued states, respectively. In Section 8 we summarize the conclusions.

\section{Basic notions}

First we recall from [4] some notions of universal algebra that will play an important role in what follows. A variety is a class of algebras of the same type defined by a set of equations. Let $\mathcal{A}$ be a variety of algebras of type $\sigma$. We denote by Term $_{\mathcal{A}}$ the absolutely free algebra of type $\sigma$ built from the set of variables $V=\left\{x_{1}, x_{2}, \ldots\right\}$. Each element of $\operatorname{Term}_{\mathcal{A}}$ is referred to as a term. We denote by $\operatorname{Comp}(t)$ the complexity of the term $t$ and by $t=s$ the equations of $\operatorname{Term}_{\mathcal{A}}$. Let $A \in \mathcal{A}$. If $t \in \operatorname{Term}_{\mathcal{A}}$ and $a_{1}, \ldots, a_{n} \in$ $A$, by $t^{A}\left(a_{1}, \ldots, a_{n}\right)$ we denote the result of the application of the term operation $t^{A}$ to the elements $a_{1}, \ldots, a_{n}$. A valuation in $A$ is a map $v: V \rightarrow$ $A$. Of course, any valuation $v$ in $A$ can be uniquely extended to an $\mathcal{A}$ homomorphism $v: \operatorname{Term}_{\mathcal{A}} \rightarrow A$ in the usual way, i.e., if $t_{1}, \ldots, t_{n} \in \operatorname{Term}_{\mathcal{A}}$ then $v\left(t\left(t_{1}, \ldots, t_{n}\right)\right)=t^{A}\left(v\left(t_{1}\right), \ldots, v\left(t_{n}\right)\right)$. Thus, valuations are identified with $\mathcal{A}$-homomorphisms from the absolutely free algebra. If $t, s \in \operatorname{Term}_{\mathcal{A}}$, $\models_{A} t=s$ means that for each valuation $v$ in $A, v(t)=v(s)$ and $\models_{\mathcal{A}} t=s$ means that for each $A \in \mathcal{A}, \models_{A} t=s$.

For each algebra $A \in \mathcal{A}$, we denote by $\operatorname{Con}(A)$, the congruence lattice of $A$, the diagonal congruence is denoted by $\Delta$ and the largest congruence $A^{2}$ is denoted by $\nabla$. $\theta$ is called factor congruence iff there is a congruence $\theta^{*}$ on $A$ such that, $\theta \wedge \theta^{*}=\Delta, \theta \vee \theta^{*}=\nabla$ and $\theta$ permutes with $\theta^{*}$. If $\theta$ and $\theta^{*}$ is a pair of factor congruences on $A$ then $A \cong A / \theta \times A / \theta^{*}$. $A$ is directly indecomposable if $A$ is not isomorphic to a product of two non trivial algebras or, equivalently, $\Delta, \nabla$ are the only factor congruences in $A$. We say that $A$ is subdirect product of a family of $\left(A_{i}\right)_{i \in I}$ of algebras if there exists an embedding $f: A \rightarrow \prod_{i \in I} A_{i}$ such that $\pi_{i} f: A \rightarrow A_{i}$ is a surjective homomorphism for each $i \in I$ where $\pi_{i}$ is the projection onto $A_{i}$. A is subdirectly irreducible iff $A$ is trivial or there is a minimum congruence in $\operatorname{Con}(A)-\Delta$. It is clear that a subdirectly irreducible algebra is directly 
indecomposable. An important result due to Birkhoff is that every algebra $A$ is subdirect product of subdirectly irreducible algebras. Thus the class of subdirectly irreducible algebras rules the valid equations in the variety $\mathcal{A}$.

Now we recall from [14] and [19] some notions about orthomodular lattices. Let $\langle P, \leq\rangle$ be a poset and $X \subseteq P$. Then $X$ is said to be increasing set iff, $a \in X$ and $a \leq x$ implies $x \in X$. A lattice with involution [13] is an algebra $\langle L, \vee, \wedge, \neg\rangle$ such that $\langle L, \vee, \wedge\rangle$ is a lattice and $\neg$ is a unary operation on $L$ that fulfills the following conditions: $\neg \neg x=x$ and $\neg(x \vee y)=\neg x \wedge \neg y$. An orthomodular lattice is an algebra $\langle L, \wedge, \vee, \neg, 0,1\rangle$ of type $\langle 2,2,1,0,0\rangle$ that satisfies the following conditions:

1. $\langle L, \wedge, \vee, \neg, 0,1\rangle$ is a bounded lattice with involution,

2. $x \wedge \neg x=0$.

3. $x \vee(\neg x \wedge(x \vee y))=x \vee y$

We denote by $\mathcal{O} \mathcal{M L}$ the variety of orthomodular lattices.

Remark 1.1 An important characterization of the equations in $\mathcal{O M L}$ is given by:

$$
\models_{\mathcal{O} \mathcal{M L}} t=s \quad \text { iff } \quad \models_{\mathcal{O} \mathcal{M L}}(t \wedge s) \vee(\neg t \wedge \neg s)=1
$$

Therefore we can safely assume that all $\mathcal{O} \mathcal{M L}$-equations are of the form $t=$ 1 , where $t \in \operatorname{Term}_{\mathcal{O M} \mathcal{L}}$. It is clear that this characterization is maintained for each variety $\mathcal{A}$ such that there are terms of the language of $\mathcal{A}$ defining on each $A \in \mathcal{A}$ operations $\vee, \wedge, \neg, 0,1$ such that $L(A)=\langle A, \vee, \wedge, \neg, 0,1\rangle$ is an orthomodular lattice.

Let $L$ be an orthomodular lattice. Two elements $a, b$ in $L$ are orthogonal (noted $a \perp b$ ) iff $a \leq \neg b$. For each $a \in L$ let us consider the interval $[0, a]=$ $\{x \in L: 0 \leq x \leq a\}$ and the unary operation in $[0, a]$ given by $\neg a x=x^{\prime} \wedge a$. As one can readily realize, the structure $L_{a}=\left\langle[0, a], \wedge, \vee, \neg_{a}, 0, a\right\rangle$ is an orthomodular lattice.

Boolean algebras are orthomodular lattices satisfying the distributive law $x \wedge(y \vee z)=(x \wedge y) \vee(x \wedge z)$. We denote by $\mathbf{2}$ the Boolean algebra of two elements. Let $A$ be a Boolean algebra. A subset $F$ of $A$ is called a filter iff it is an increasing set and, if $a, b \in F$ then $a \wedge b \in F$. F is a proper filter iff $F \neq A$ or, equivalently, $0 \notin F$. For each $a>0,[a)=\{x \in L: a \leq x\}$ is a filter called principal filter generated by a. Each filter $F$ in $A$ determines 
univocally a congruence $\theta_{F}$ and viceversa. In this case the quotient set $A / \theta_{F}$, noted as $A / F$, is a Boolean algebra and the natural application $x \mapsto[x]$ is a Boolean homomorphism from $A$ to $A / F$. It may be easily proved that each filter in $A$ determines a factor congruence, thus the unique directly indecomposable Boolean algebra is $\mathbf{2}$. A proper filter $F$ is maximal iff the quotient algebra $A / F$ is isomorphic to $\mathbf{2}$ iff $x \notin F$ implies $\neg x \in F$. It is well known that each proper filter can be extended to a maximal one.

Let $L$ be an orthomodular lattice. An element $c \in L$ is said to be a complement of $a$ iff $a \wedge c=0$ and $a \vee c=1$. Given $a, b, c$ in $L$, we write: $(a, b, c) D$ iff $(a \vee b) \wedge c=(a \wedge c) \vee(b \wedge c) ;(a, b, c) D^{*}$ iff $(a \wedge b) \vee c=(a \vee c) \wedge(b \vee c)$ and $(a, b, c) T$ iff $(a, b, c) D,(\mathrm{a}, \mathrm{b}, \mathrm{c}) D^{*}$ hold for all permutations of $a, b, c$. An element $z$ of $L$ is called central iff for all elements $a, b \in L$ we have $(a, b, z) T$. We denote by $Z(L)$ the set of all central elements of $L$ and it is called the center of $L$.

Proposition 1.2 Let $L$ be an orthomodular lattice. Then we have:

1. $Z(L)$ is a Boolean sublattice of $L[19$, Theorem 4.15].

2. $z \in Z(L)$ iff for each $a \in L, a=(a \wedge z) \vee(a \wedge \neg z)$ [19, Lemma 29.9].

\section{The relevance of two-valued states}

In general, two-valued states associated to a quantum system are probability measures $s: E \rightarrow\{0,1\}$ where $E$ is a set equipped with an orthostructure called event structure. The study of the different families of two-valued states becomes relevant in different frameworks.

From a physical point of view, two-valued measures are distinguished among the set of all classes of states because of their relation to hidden variable theories of quantum mechanics. The discussion about the necessity of adding hidden variables (HV) to standard physical magnitudes in quantum mechanics (QM) in order to provide a complete account of physical reality began with the famous so called EPR paper [8] which Einstein and his students Podolsky and Rosen presented in 1935. At the end of the paper, they state that "While we have [thus] shown that the wave function does not provide a complete description of the physical reality, we left open the question of whether or not such a description exists. We believe, however, 
that such a theory is possible." A possible reading of the EPR conclusion was endorsed by the HV program which attempted to complete the quantum description with hidden magnitudes which would allow, at least in principle, to predict with certainty the results of observations. Against such attempts, von Neumann developed a theorem which seemed to preclude $\mathrm{HV}$ due to the inexistence of dispersion free states (DFS, i.e. states for which $<\mathbf{A}>^{2}=<\mathbf{A}^{2}>$ ) compatible with the mathematical structure of the theory [23, pg. 232]. Von Neumann considered the measurement of a physical magnitude over an ensemble of systems in the same state. QM predicts that, in the general case, each measurement will give as a result any of the eigenvalues of the operator representing the magnitude. Thus, although all the systems are in the same state, we obtain different results for the measurement of the same quantity. According to von Neumann, this is so either because there are some HV which the quantum description does not take into account or because, though the systems are really in the same state, the dispersion of measured values is due to Nature itself. If QM were to be described by HV, the ensemble would have to contain as many sub-ensembles as there are different eingevalues, with every system in a sub-ensemble in a DFS characterized by a particular value of each $\mathrm{HV}$. Starting from a set of assumptions he considered plausible, von Neumann proved that the usual Hilbert space model for QM does not admit HV. Jauch and Piron $[12,25]$ have shown that the same result holds when taking into account more general models. However, Bohmian mechanics [3] seemed to fragrantly contradict von Neumann's theorem, thus opening the analysis of the strength of the hypothesis and presuppositions involved in the theorems. Observing this anomaly, Bell reconsidered the HV program. Bell believed that "[...] quantum mechanics could not be a complete theory but should be complemented by additional variables. These additional variables were to restore to the theory causality and locality." [1, pg. 195]. Bell wanted to show the possibility of, in principle, completing QM with HV. But contrary to his own expectations he himself proved, developing a by now famous inequality, that no local, realistic HV theory would be able to reproduce the statistical predictions of QM. Bohmian mechanics could do so at the price of giving up locality.

Bell's theorem proves that, in order to keep alive the HV program, either some physical presupposition had to be given up or at least some part of the formalism had to be changed. The latter possibility allows to develop various HV theories, each one based on a particular family of two-valued states, as described in [11, Ch. 4]. In fact, considering a family of two-valued states 
called dispersion free and some hypothesis on the event structure it is possible to define a theory of $\mathrm{HV}$ in the von Neumann style in which the only event structures that admit $\mathrm{HV}$ are classical structures (see [11, Theorem 3.24]). However, the requirement of classicality may be circumvented developing a HV theory based on probability weakening the hypothesis over the mentioned family of two-valued states and imposing certain restrictions on the orthostructure of the event space (see [11, Theorem 3.26]).

Another motivation for the analysis of various families of two-valued states is rooted in the study of algebraic and topological representations of the event structures. These results give rise to a new mathematical description of quantum systems. Examples of them are the characterization of Boolean orthoposets by means of two-valued states [34] and the representation of orthomodular lattices via clopen sets in a compact Hausdorff closure space [33], later extended to orthomodular posets in [18].

In the above mentioned cases, the family of two-valued states is conceived as an "external object" to the event structure in the following sense: given a class of event structures $\mathcal{E}$ and a family of two-valued probability measures, it is of interest to know which events $E \in \mathcal{E}$ admit such probability measures. As mentioned in the introduction, our aim is to "internalize" the concept of two-valued state by enlarging the event structure with a unary operation. From a conceptual point of view, this approach would allow to consider the possible theories of $\mathrm{HV}$ based on two-valued states as interior objects in the event structure. In other words, an event structure expanded by an

operation that defines a family of two-valued states would determine in some sense its own family of $\mathrm{HV}$ theories.

\section{Boolean pre-states on orthomodular lattices}

We formally present here the notion of two-valued state over orthomodular lattices. Let $L$ be an orthomodular lattice.

Definition 3.1 A two-valued state on $L$ is a function $\sigma: L \rightarrow\{0,1\}$ such that:

1. $\sigma(1)=1$,

2. if $x \perp y$ then $\sigma(x \vee y)=\sigma(x)+\sigma(y)$.

Consider the set $\{0,1\}$ equipped with the usual Boolean structure. As we will show in detail from Section 6 on, the different classes of two-valued 
states are functions from an orthomodular lattice $L$ onto the set $\{0,1\}$ that preserve the orthostructure, i.e., order and orthocomplementation. These properties are very important since they rule certain algebraic characteristics which are common to different classes of two valued states. This observation motivates the following general definition:

Definition 3.2 Let $L$ be an orthomodular lattice. By a Boolean pre-state on $L$ we mean a function $\sigma: L \rightarrow\{0,1\}$ such that:

1. $\sigma(\neg x)=1-\sigma(x)$,

2. if $x \leq y$ then $\sigma(x) \leq \sigma(y)$.

We denote by $\mathcal{E}_{B}$ the category whose objects are pairs $(L, \sigma)$ such that $L$ is an orthomodular lattice and $\sigma$ is a Boolean pre-state on $L$. Arrows in $\mathcal{E}_{B}$ are $\left(L_{1}, \sigma_{1}\right) \stackrel{f}{\rightarrow}\left(L_{2}, \sigma_{2}\right)$ such that $f: L_{1} \rightarrow L_{2}$ is a $\mathcal{O} \mathcal{M L}$-homomorphism, and the following diagram is commutative:

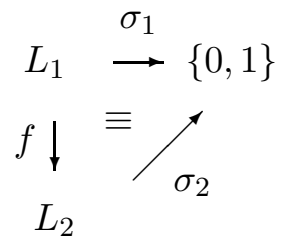

These arrows are called $\mathcal{E}_{B}$-homomorphisms. The following proposition is immediate.

Proposition 3.3 Let $L$ be an orthomodular lattice and $\sigma$ a Boolean prestate on $L$. Then:

1. $\sigma(1)=1$ and $\sigma(0)=0$,

2. $\sigma(x \wedge y) \leq \min \{\sigma(x), \sigma(y)\}$,

The basic properties of the Boolean pre-states and the notion of $\mathcal{E}_{B^{-}}$ homomorphisms suggest that Boolean pre-states can be seen as new unary operations that expand the orthomodular structure. This motivates the following definition: 
Definition 3.4 An orthomodular lattice with an internal Boolean pre-state (IE $E_{B}$-lattice for short) is an algebra $\langle L, \wedge, \vee, \neg, s, 0,1\rangle$ of type $\langle 2,2,1,1,0,0\rangle$ such that $\langle L, \wedge, \vee, \neg, 0,1\rangle$ is an orthomodular lattice and $s$ satisfies the following equations for each $x, y \in A$ :

s1. $s(1)=1$.

s2. $s(\neg x)=\neg s(x)$,

s3. $s(x \vee s(y))=s(x) \vee s(y)$,

s4. $y=(y \wedge s(x)) \vee(y \wedge \neg s(x))$,

s5. $s(x \wedge y) \leq s(x) \wedge s(y)$.

We shall refer to $s$ as a internal Boolean pre-state. Clearly Axiom s5 may be equivalently formulated as the equation $s(x \wedge y)=s(x \wedge y) \wedge(s(x) \wedge s(y))$. Thus, the class of $I E_{B}$-lattices is a variety that we call $\mathcal{I} \mathcal{E}_{B}$.

Let $L_{1}$ and $L_{2}$ be two $I E_{B}$-lattices. $f: L_{1} \rightarrow L_{2}$ is a $\mathcal{I} \mathcal{E}_{B}$-homomorphism iff it is an $\mathcal{O} \mathcal{M L}$-homomorphism and $f(s(x))=s(f(x))$ for each $x \in A$. Note that $\mathcal{I E}_{B}$-homomorphisms have analog properties to those of arrows in the category $\mathcal{E}_{B}$. Let $\mathcal{A}$ be a subvariety of $\mathcal{I} \mathcal{E}_{B}$. Since $\mathcal{A}$ admits an orthomodular reduct, all the equations in $\mathcal{A}$ can be referred to 1 . Moreover, $\mathcal{A}$ is an arithmetical variety, i.e. it is both congruence-distributive and congruencepermutable. The following Proposition provides the main properties of $I E_{B^{-}}$ lattices.

Proposition 3.5 Let $L$ be a $I E_{B}$-lattice. Then we have:

1. $\langle s(L), \vee, \wedge, \neg, 0,1\rangle$ is a Boolean sublattice of $Z(L)$,

2. If $x \leq y$ then $s(x) \leq s(y)$,

3. $s(x) \vee s(y) \leq s(x \vee y)$,

4. $s(s(x))=s(x)$,

5. $x \in s(L)$ iff $s(x)=x$,

6. $s(x \wedge s(y))=s(x) \wedge s(y)$. 
Proof: 1) Let $x \in S(L)$. Then there exists $x_{0} \in L$ such that $x=s\left(x_{0}\right)$. By s4, $y=\left(y \wedge s\left(x_{0}\right)\right) \vee\left(y \wedge \neg s\left(x_{0}\right)\right)=(y \wedge x) \vee(y \wedge \neg x)$ for each $y \in L$. Therefore, by Proposition 1.2-2, $x \in Z(L)$ and $s(L) \subseteq Z(L)$. By s1, s2 and s3, note that 0,1 lie in $s(L), \neg$ and $\vee$ are closed operations in $s(L)$. Hence $\langle s(L), \vee, \wedge, \neg, 0,1\rangle$ is a Boolean sublattice of $Z(L)$.

2) Suppose that $x \leq y$. Then $s(x)=s(x \wedge y) \leq s(x) \wedge s(y)$. Thus $s(x)=s(x) \wedge s(y)$ and $s(x) \leq s(y)$. 3) Follows from item 2. 4) By s3, $s(s(x))=s(0 \vee s(x))=s(0) \vee s(x)=0 \vee s(x)=s(x)$. 5$)$ If $x \in s(L)$ then there exists $x_{0} \in L$ such that $x=s\left(x_{0}\right)$. Therefore, by item $4, s(x)=s\left(s\left(x_{0}\right)\right)=s\left(x_{0}\right)=x$. $\quad$ 6) $s(x \wedge s(y))=\neg s(\neg x \vee s(\neg y))=$ $\neg(\neg s(x) \vee \neg s(y))=s(x) \wedge s(y)$.

Let $L$ be an orthomodular lattice. An element $a$ is said to be perspective to $b$ (noted $a \sim b$ ) iff $a$ and $b$ have a common complement, i.e. there exists $x \in L$ such that $a \vee x=1=b \vee x$ and $a \wedge x=0=b \wedge x$. An OML-filter (also called perspective filter [14]) in $L$ is a subset $F \subseteq A$ that satisfies the following conditions:

1. $F$ is an increasing set,

2. if $a, b \in F$ then $a \wedge b \in F$,

3. if $a \in F$ and $a \sim b$ then $b \in F$.

We denote by Filt $(L)$ the complete lattice of $O M L$-filters in $L$. If we define the map $\operatorname{Con}(L) \ni \theta \mapsto \alpha(\theta)=\{x \in L:(x, 1) \in \theta\}$ then $\alpha$ provides a lattice isomorphism from $\operatorname{Con}(L)$ onto $F_{O M L}(L)$ whose inverse is given by $\alpha^{-1}(F)=\left\{(x, y) \in L^{2}:(x \wedge y) \vee(\neg x \wedge \neg y) \in F\right\}$ for each $F \in F_{O M L}(L)$ $[14, \S 2$ Theorem 6].

Definition 3.6 Let $L$ be a $I E_{B}$-lattice. A $I E_{B}$-filter in $L$ is an $O M L$-filter of $L$ which is closed under $s$.

Let $L$ be a $I E_{B}$-lattice. We denote by $F_{i l t} E_{B}(L)$ the set of all $I E_{B^{-}}$ filters in $L$ and by $\operatorname{Con}_{I E_{B}}(L)$ the congruences lattice of $L$. Clearly Filt $_{I E_{B}}(L)$ is a complete lattice. Given a congruence $\theta \in \operatorname{Con}_{I E_{B}}(L)$, we define:

$$
F_{\theta}=\{x \in L:(x, 1) \in \theta\}
$$

Conversely, given $F \in$ Filt $_{I E_{B}}(L)$ we define:

$\theta_{F}=\left\{(x, y) \in L^{2}:(x \wedge y) \vee(\neg x \wedge \neg y) \in F\right.$ and $\left.s(x \wedge y) \vee s(\neg x \wedge \neg y) \in F\right\}$ 
Theorem 3.7 Let $L$ be a $I E_{B}$-lattice. The maps $F \mapsto \theta_{F}$ and $\theta \mapsto F_{\theta}$ are mutually inverse lattice-isomorphisms between $\operatorname{Con}_{I E_{B}}(L)$ and Filt $_{I E_{B}}(L)$.

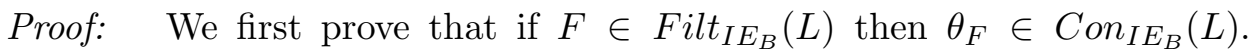
By definition it is clear that $\theta_{F}$ is an $O M L$-congruence. Thus we have to prove that $\theta_{F}$ is $s$-compatible. Let $(x, y) \in \theta_{F}$. By Axiom s5 we have $F \ni s(x \wedge y) \vee s(\neg x \wedge \neg y) \leq(s(x) \wedge s(y)) \vee(\neg s(x) \wedge \neg s(y))$ and then:

$$
(s(x) \wedge s(y)) \vee(\neg s(x) \wedge \neg s(y)) \in F
$$

By s3, Proposition 3.5 and taking into account that $F$ is closed by $s$ we have: $s((s(x) \wedge s(y)) \vee(\neg s(x) \wedge \neg s(y))) \in F$ and

$$
\begin{aligned}
s((s(x) \wedge s(y)) \vee(\neg s(x) \wedge \neg s(y))) & =s((s(x) \wedge s(y)) \vee s(\neg x \wedge s(\neg y))) \\
& =s(s(x) \wedge s(y)) \vee s(s(\neg x \wedge s(\neg y))) \\
& =s(s(x) \wedge s(y)) \vee s(\neg s(x) \wedge \neg s(y))
\end{aligned}
$$

Hence,

$$
s(s(x) \wedge s(y)) \vee s(\neg s(x) \wedge \neg s(y)) \in F
$$

Thus $(s(x), s(y)) \in \theta_{F}$, i.e. $\theta_{F}$ is $s$-compatible and $\theta_{F} \in \operatorname{Con}_{I E_{B}}(L)$.

For the converse, suppose that $\theta_{F} \in \operatorname{Con}_{I E_{B}}(L)$. Since $\theta_{F}$ is a $O M L$ congruence, $F=\left\{x \in L:(x, 1) \in \theta_{F}\right\}$ is $O M L$-filter. Since $s(1)=1$, $F$ is closed by $s$ and then $F \in F_{i l t} I_{B}(L)$. Since the maps $F \mapsto \theta_{F}$ and $\theta \mapsto F_{\theta}$ are mutually inverse lattice-isomorphisms between $\operatorname{Con}_{O M L}(L)$ and

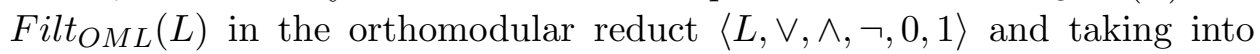
account that $F \in$ Filt $_{I E_{B}}(L)$ iff $\theta_{F} \in \operatorname{Con}_{I E_{B}}(L)$, we have that $F_{i l t_{I E_{B}}}(L)$ and $\operatorname{Con}_{I E_{B}}(L)$ are lattice-order isomorphic.

\section{The functor $\mathcal{I}$}

In this section we show that starting from a $I E_{B}$-lattice $L$, it is possible to define Boolean pre-states on the underling orthomodular structure of $L$. This operation gives rise to a functor from the category of $I E_{B}$-lattices onto the category of Boolean pre-states. We first introduce some basic notions.

Definition 4.1 Let $B$ be a Boolean algebra. An increasing subset $M \subseteq B$ is said to be prime iff it satisfies: $x \in M$ iff $\neg x \notin M$.

Proposition 4.2 Let $B$ be a Boolean algebra. Then for each a $>0$ there exists a prime increasing subset $M$ of $B$ such that $a \in M$. 
Proof: $\quad$ Clearly if $x \in[a)$ then $\neg x \notin[a)$. By Zorn's Lemma there exists a maximal increasing set $M$ such that, $[a) \subseteq M$ and, $x \in M$ implies $\neg x \notin M$. Suppose that $x, \neg x \notin M$. Let $M_{1}=M \cup[x)$. Clearly $M_{1}$ is an increasing set. We will show that if $y \in M_{1}$ then $\neg y \notin M_{1}$. If $y \in M_{1}$ we have to consider two cases:

case 1: $y \in M$. In this case $\neg y \notin M$. If $\neg y \in[x)$ then $x \leq \neg y, y \leq \neg x$ and $\neg x \in M$ which is a contradiction. Thus $\neg y \notin M_{1}$.

case 2: $y \in[x)$. Then $x \leq y$ and $\neg y \notin[x)$. Moreover $\neg y \leq \neg x$. If $\neg y \in M$ then $\neg x \in M$ which is a contradiction. Thus $\neg y \notin M \cup[x)$.

Hence $\neg y \notin M_{1}$. Since $M$ is a maximal increasing set respect to the property $x \in M$ implies $\neg x \notin M$, we have that $M=M_{1}=M \cup[x)$ which is a contradiction since $x, \neg x \notin M$. This proves that, if $\neg x \notin M$ then $x \in M$. Thus $M$ satisfies the property $x \in M$ iff $\neg x \notin M$ and then $M$ is a prime increasing subset of $B$ containing $a$.

Proposition 4.3 Let $B$ be a Boolean algebra and $\sigma$ be Boolean pre-state on $B$. Then the map $\sigma \mapsto \sigma^{-1}(1)=\{x \in B: \sigma(x)=1\}$ is a one-to-one correspondence between Boolean pre-states on $B$ and prime increasing subset of $B$.

Proof: Since $\sigma$ is an order homomorphism then $\sigma^{-1}$ is an increasing set. Moreover $x \in \sigma^{-1}(1)$ iff $\sigma(x)=1$ iff $\sigma(\neg x)=0$ iff $\neg x \notin \sigma^{-1}(1)$. Thus $\sigma^{-1}(1)$ is prime increasing subset of $B$. By definition, the map $\sigma \mapsto \sigma^{-1}(1)$ is injective. We prove the surjectivity. Let $M$ be a prime increasing subset of $B$. If we consider the function

$$
\sigma_{M}(x)= \begin{cases}1, & \text { if } x \in M \\ 0, & \text { otherwise }\end{cases}
$$

it is not very hard to see that $\sigma_{M}$ is Boolean pre-state and $\sigma_{M}^{-1}(1)=M$. Hence the map is surjective.

Proposition 4.4 Let $L$ be a $I E_{B}$-lattice. Then there exists a Boolean prestate $\sigma: L \rightarrow\{0,1\}$ such that $\sigma(x)=1$ iff $\sigma(s(x))=1$.

Proof: By Proposition 4.2, there exists a prime increasing subset $M$ of $s(L)$. By Proposition 4.3, let $\varphi_{M}: s(L) \rightarrow\{0,1\}$ be the Boolean pre-state associated to $M$. Define the composition $\sigma_{M}: L \stackrel{s}{\rightarrow} s(L) \stackrel{\varphi_{M}}{\rightarrow}\{0,1\}$. Clearly 
$\sigma_{M}$ is an order homomorphism and note that $\sigma_{M}(\neg x)=\varphi_{M}(s(\neg x))=$ $\varphi_{M}(\neg s(x))=1-\varphi_{M}(s(x))$. Hence $\sigma_{M}$ is a Boolean pre-state on $L$. By Proposition 3.5-4, $\sigma_{M}(x)=1$ iff $1=\varphi_{M}(s(x))=\varphi_{M} s(s(x))=\sigma_{M}(s(x))$.

The last proposition motivates the following concept:

Definition 4.5 Let $L$ be a $I E_{B}$-lattice and $\sigma$ be a Boolean pre-state on $L$. Then $s, \sigma$ are coherent whenever they satisfy: $\sigma(x)=1$ iff $\sigma(s(x))=1$.

Proposition 4.4 allows to build a coherent Boolean pre-state for each possible prime increasing set in $s(L)$. Our main interest is to tell exactly if all possible Boolean pre-states in $L$, coherent with $s$, come from a prime increasing set in $s(L)$. In order to do this, we extend the concept of prime increasing subset to the $I E_{B}$-lattices in the following manner:

Definition 4.6 Let $L$ be a $I E_{B}$-lattice. A Boolean pre-state filter (bps-filter for short) is a non-empty subset $F$ of $L$ such that

1. $F$ is an increasing set such that $s(F) \subseteq F$,

2. $x \in F$ iff $\neg x \notin F$

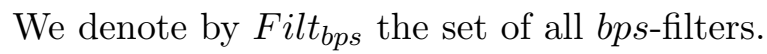

Lemma 4.7 Let $L$ be a $I E_{B}$-lattice and $F$ be a bps-filter. Then $s(F)$ is a prime increasing subset in $s(L)$.

Proof: $\quad$ Let $a \in s(F)$ and $x \in s(L)$ such that $a \leq x$. By definition of bps-filter, $s(F) \subseteq F$ and then $a \in F$. Since $F$ is an increasing set, $x \in F$.

By Proposition 3.5-5, $x=s(x) \in s(F)$ and then $s(F)$ is an increasing set in $s(L)$. Let $x \in s(L)$. Since $x=s(x)$ and $F$ is closed by $s$, we have: $x \in s(F)$ iff $x \in F$ iff $\neg x \notin F$ iff $\neg x \notin s(F)$. Hence $s(F)$ is a prime increasing subset in $s(L)$.

Proposition 4.8 Let $L$ be a $I E_{B}$-lattice and $M$ be a prime increasing subset in $s(L)$. Then the map $M \mapsto F_{M}=\{x \in L: s(x) \in M\}$ is a one-to-one correspondence between prime increasing subsets in $s(L)$ and Filt $_{\text {bps }}(L)$. 
Proof: By Proposition 3.5-2, $F_{M}$ is an increasing set. For each $x \in F_{M}$, $s(x) \in M$ and then $s(x)=s(s(x)) \in M$. Thus $s(x) \in F_{M}$ and $F_{M}$ is closed by $s$. Let $x \in L$. Then $x \in F_{M}$ iff $s(x) \in M$ iff $\neg s(x) \notin M$ iff $\neg x \notin F_{M}$. Hence $F_{M} \in$ Filt $_{\text {bps }}(L)$. By definition it is not very hard to see that the map $M \mapsto F_{M}=\{x \in L: s(x) \in M\}$ is injective. We shall prove the surjectivity. Let $F \in$ Filt $_{b p s}(L)$. By Lemma 4.7, $s(F)$ is a prime increasing subset in $s(L)$. By the above result we can consider the bps-filter $F_{s(F)}$. If $x \in F_{s(F)}$ then $s(x) \in s(F)$. Note that if $x \notin F$ then $\neg x \in F$ and $\neg s(x) \in s(F)$ which is a contradiction. Therefore $x \in F$ and $F_{s(F)} \subseteq F$. For the other inclusion, if $x \in F$ then $s(x) \in s(F)$ and $x \in F_{s(F)}$. Thus $F \subseteq F_{s(F)}$. Hence $F=F_{s(F)}$. These arguments prove that $M \mapsto F_{M}=\{x \in L: s(x) \in M\}$ is a one-to-one correspondence between prime increasing subsets in $s(L)$ and $F_{i l t}$ bps $(L)$.

Proposition 4.9 Let $L$ be a $I E_{B}$-lattice and $\left(\sigma_{i}\right)_{i}$ be the family of Boolean pre-states on $L$ coherent with $s$. Then there exists a one-to-one correspondence between $\left(\sigma_{i}\right)_{i}$ and Filt bps $_{(}(L)$ given by the mapping $\sigma_{i} \mapsto \sigma_{i}^{-1}(1)$.

Proof: We first prove that if $\sigma$ is a Boolean pre-state on $L$ coherent with $s$ then $\sigma^{-1}(1)$ is a bps-filter. Clearly $\sigma^{-1}(1)$ is an increasing set. Since $\sigma$ is coherent with $s$ then $\sigma^{-1}(1)$ is closed by $s . x \in \sigma^{-1}(1)$ iff $\sigma(x)=1$ iff $\sigma(\neg x)=0$ iff $\neg x \notin \sigma^{-1}(1)$. Hence $\sigma^{-1}(1) \in$ Filt $_{\text {bps }}(L)$. Trivially the map $\sigma_{i} \mapsto \sigma_{i}^{-1}(1)$ is injective. Then we have to prove the surjectivity.

Let $F \in F_{\text {ilt }}$ bps $(L)$. By Lemma 4.7, $s(F)$ is a prime increasing subset of $s(L)$. With the same argument used in Proposition 4.4, consider the Boolean pre-state $\sigma_{s(F)}$ coherent with $s$ given by the composition $L \stackrel{s}{\rightarrow}$ $s(L) \stackrel{\varphi_{s(F)}}{\rightarrow}\{0,1\}$. We have to prove that $F=\sigma_{s(F)}^{-1}(1)$. If $x \in \sigma_{s(F)}^{-1}(1)$ then $\varphi_{s(F)}(s(x))=1$. Therefore $s(x) \in s(F)$. Suppose that $x \notin F$. Since $F$ is a bps-filter, $\neg x \in F$ and $\neg s(x) \in s(F)$ which is a contradiction since $s(F)$ is a prime increasing subset on $s(L)$. Thus $x \in F$ and $\sigma_{s(F)}^{-1}(1) \subseteq F$. On the other hand, if $x \in F$ then, $s(x) \in s(F)$ and $\sigma_{s(F)}(x)=\varphi_{s(F)}(s(x))=1$. Thus $x \in \sigma_{s(F)}^{-1}(1)$ and $F \subseteq \sigma_{s(F)}^{-1}(1)$.

Thus, by Propositions 4.8 and 4.9 , for a $I E_{B}$-lattice $L$, Boolean prestates on $L$ are in one-to-one correspondence with prime increasing sets in $s(L)$. Moreover, we can built $I E_{B}$-lattices from an object in the category $\mathcal{E}_{B}$. As we shall see in the following, this construction is described by a functor. 
Proposition 4.10 Let $L$ be an orthomodular lattice and $\sigma$ be a Boolean pre-state on $L$. If we define $\mathcal{I}(L)=\left(L, s_{\sigma}\right)$ such that

$$
s_{\sigma}(x)= \begin{cases}1^{L}, & \text { if } \sigma(x)=1 \\ 0^{L}, & \text { if } \sigma(0)=0\end{cases}
$$

then:

1. $\mathcal{I}(L)$ is a $I E_{B}$-lattice and $s_{\sigma}$ is coherent with $\sigma$.

2. If $\sigma(x \vee y)=\sigma(x)+\sigma(y)$ then $s_{\sigma}(x \vee y)=s_{\sigma}(x) \vee s_{\sigma}(y)$.

3. If $\left(L_{1}, \sigma_{1}\right) \stackrel{f}{\rightarrow}\left(L_{2}, \sigma_{2}\right)$ is a $\mathcal{E}_{B}$-homomorphism then $f: \mathcal{I}\left(L_{1}\right) \rightarrow \mathcal{I}\left(L_{2}\right)$ is a $I E_{B}$-homomorphism.

Proof: 1) We have to prove that $s_{\sigma}$ satisfies $\mathrm{s} 1, \ldots, \mathrm{s} 5$. Clearly s1, s2 and s4 are trivially satisfied. s3) If $\sigma(y)=1$ then, $1^{L}=s_{\sigma}\left(x \vee 1^{L}\right)=s_{\sigma}\left(x \vee s_{\sigma}(y)\right)$ and $s_{\sigma}(x) \vee s_{\sigma}(y)=s_{\sigma}(x) \vee 1^{L}=1^{L}$. If $\sigma(y)=0$ then $s_{\sigma}\left(x \vee s_{\sigma}(y)\right)=s_{\sigma}(x)$ and $s_{\sigma}(x) \vee s_{\sigma}(y)=s_{\sigma}(x)$. $\quad$ s5) If $\sigma(x \wedge y)=0$ then $s_{\sigma}(x \wedge y)=0$ and $0^{L}=s_{\sigma}(x \wedge y) \leq s_{\sigma}(x) \wedge s_{\sigma}(y)$. Suppose that $\sigma(x \wedge y)=1$. Since $\sigma$ is monotone $\sigma(x)=\sigma(y)=1$. Thus $s_{\sigma}(x \wedge y)=s_{\sigma}(x) \wedge s_{\sigma}(y)$. Hence $L$ with the operation $s_{\sigma}$ is a $I E_{B}$-lattice. Note that $\sigma(x)=1$ iff $s_{\sigma}(x)=1^{L}$ iff $\sigma\left(s_{\sigma}(x)\right)=1$ and then $s_{\sigma}$ is coherent with $\sigma$.

2 ) Suppose that $\sigma(x)=1$. Then $1^{L}=s_{\sigma}(x) \leq s_{\sigma}(x) \vee s_{\sigma}(y)$. Since $x \leq x \vee y$, we have $\sigma(x \vee y)=1$ and $s_{\sigma}(x \vee y)=1^{L}$. Thus $s_{\sigma}(x \vee y)=$ $s_{\sigma}(x) \vee s_{\sigma}(y)=1^{L}$. The case $\sigma(y)=1$ is analogous. Suppose that $\sigma(x)=$ $\sigma(y)=0$. Then $s_{\sigma}(x) \vee s_{\sigma}(y)=0^{L}$. Moreover $\sigma(x \vee y)=\sigma(x)+\sigma(y)=0$ and $0^{L}=s_{\sigma}(x \vee y)$. Thus $s_{\sigma}(x \vee y)=s_{\sigma}(x) \vee s_{\sigma}(y)=0^{L}$.

3) Let $\left(L_{1}, \sigma_{1}\right) \stackrel{f}{\rightarrow}\left(L_{2}, \sigma_{2}\right)$ is a $\mathcal{E}_{B}$-homomorphism. Suppose that $\sigma_{1}(x)=$ 1. Then $f\left(s_{\sigma_{1}}(x)\right)=f\left(1^{L_{1}}\right)=1^{L_{2}}$. Since $\sigma_{2} \circ f=\sigma_{1}, \sigma_{2}(f(x))=1$ and then $s_{\sigma_{2}}(f(x))=1^{L_{2}}$. An analogous result can be obtained when we consider the case $\sigma_{1}(x)=0$. Hence $f\left(s_{\sigma_{1}}(x)\right)=s_{\sigma_{2}}(f(x))$.

By Proposition 4.10 we can see that:

$$
\mathcal{I}: \mathcal{E}_{B} \rightarrow \mathcal{I E}_{B}
$$

such that $\mathcal{E}_{B} \ni(L, \sigma) \mapsto \mathcal{I}(L, \sigma)=\left(L, s_{\sigma}\right)$ and $\mathcal{I}(f)=f$ for each $\mathcal{I E}_{B^{-}}$ homomorphisms $f$, is a functor. 


\section{Equational characterization for subclasses of $\mathcal{E}_{B}$}

Boolean pre-states are external maps with respect to the orthomodular structure in the sense that they are not closed in the domain of definition. However, a closer look shows that the equational system of $\mathcal{I E}_{B}$ allows to represent the basic properties that define these maps by adding an operation to the orthomodular structure. Let $\mathcal{A}$ be a subcategory of $\mathcal{E}_{B}$. To find this operation, we propose to search for a subvariety $\mathcal{A}_{I}$ of $\mathcal{I E}_{B}$ and a subclass $\mathcal{D}$ of $\mathcal{A}_{I}$ whose algebras are univocally determined by the objects of $\mathcal{A}$ and then to see that the valid equations in $\mathcal{A}_{I}$ are determined by the subclass $\mathcal{D}$. This motivates the following definition:

Definition 5.1 Let $\mathcal{A}$ be a subcategory of $\mathcal{E}_{B}$. A subvariety $\mathcal{A}_{I}$ of $\mathcal{I E}_{B}$ equationally characterizes $\mathcal{A}$ iff there exists a subclass $\mathcal{D}$ of $\mathcal{A}_{I}$ such that:

1. $\mathcal{D}$ is categorically equivalent to $\mathcal{A}$,

2. $\models_{\mathcal{A}_{I}} t=1$ iff $\models_{\mathcal{D}} t=1$.

By an argument of universal algebra, for each subcategory $\mathcal{A}$ of $\mathcal{E}_{B}$, it is always possible to obtain a subvariety $\mathcal{A}_{I}$ of $\mathcal{I E}_{B}$ that equationally characterizes $\mathcal{A}$. In fact: we first consider the class $\mathcal{D}=\{\mathcal{I}(A): A \in \mathcal{A}\}$ that in turn allows to locally invert the functor $\mathcal{I}$ in $\mathcal{D}$, i.e. $\mathcal{I}: \mathcal{A} \rightarrow \mathcal{D}$ determines a categorical equivalence. Let $\mathcal{A}_{I}=\mathcal{V}(\mathcal{D})$ be the subvariety of $\mathcal{I E}_{B}$ generated by $\mathcal{D}$. Then $\models_{\mathcal{A}_{I}} t=1$ iff $\models_{\mathcal{D}} t=1$.

Clearly this construction does not seem very attractive because it would not give, in principle, any information about the equational system that defines the subvariety $\mathcal{A}_{I}$. Our proposal is to give arguments that allow to determine in the simplest form the equations that define $\mathcal{A}_{I}$ and the subclass $\mathcal{D}$. For this purpose, we need to characterize the direct indecomposable algebras in any subvariety of $\mathcal{I E}_{B}$ and the following preview results:

Proposition 5.2 Let $L$ be a IE $E_{B}$-lattice, $a \in s(L)$ and $L_{a}=[0, a]$. If we consider the restriction $s \uparrow_{[0, a]}$ then $\left(L_{a}, s \uparrow_{[0, a]}\right)$ is a IE $E_{B}$-lattice and $s\left(L_{a}\right)=L_{a} \cap s(L) \subseteq Z\left(L_{a}\right)$.

Proof: As is mentioned in the basic notions, $L_{a}$ is an orthomodular lattice. By Proposition 3.5-2 $s$ is closed in $L_{a}$ and then $s\left(L_{a}\right)=L_{a} \cap s(L) . \quad \mathrm{s} 3$ and s5) follow from the fact that $s$ is closed in $L_{a}$. s1) By Proposition 3.5-5, $s(a)=a . \quad \mathrm{s} 2)$ By Proposition 3.5-6 $s(\neg a x)=s(\neg x \wedge a)=s(\neg x \wedge s(a))=$ 
$\neg s(x) \wedge a=\neg_{a} s(x) . \quad$ s4) Let $x, y \in L_{a}$. Then $(y \wedge s(x)) \vee\left(y \wedge \neg_{a} s(x)\right)=$ $(y \wedge s(x)) \vee(y \wedge a \wedge \neg s(x))=(y \wedge s(x)) \vee(y \wedge \neg s(x))=y$. By Theorem 1.2 and s4 it follows that $s\left(L_{a}\right) \subseteq Z\left(L_{a}\right)$.

Proposition 5.3 Let $L$ be an $I E_{B}$-lattice and let $a, b \in s(L)$ such that $a<b$. If $v_{a}: \operatorname{Term}_{\mathcal{I E}_{B}} \rightarrow L_{a}$ is a valuation then there exists a valuation $v_{b}: \operatorname{Term}_{\mathcal{I E}_{B}} \rightarrow L_{b}$ such that $v_{a}(t)=a \wedge v_{b}(t)$.

Proof: $\quad$ We define $v_{b}:$ Term $\rightarrow L_{b}$ as follows: $v_{b}(0)=0, v_{b}(1)=b$, and $v_{b}(x)=v_{a}(x)$ for each variable $x$. We use induction on the complexity of terms. If $\operatorname{Comp}(t)=0$ (i.e. $t$ is a variable) the proof is trivial. Suppose that the Proposition holds for $\operatorname{Comp}(t)<n$. Let $t \in T e r m$ such that $\operatorname{Comp}(t)=n$. If $t$ is $\neg u$ then, $\operatorname{Comp}(u)<n$ and we have that $v_{a}(t)=$ $v_{a}(\neg u)=\neg_{a} v_{a}(u)=\neg_{a} v_{b}(u)=a \wedge \neg v_{b}(u)=a \wedge\left(b \wedge \neg v_{b}(u)\right)=a \wedge \neg_{b} v_{b}(u)=$ $a \wedge v_{b}(\neg u)=a \wedge v_{b}(t)$. Suppose that $t$ is $s(u)$. Since $\operatorname{Comp}(u)<n, s$ is closed in $L_{a}$ and $a \in s(L)$, By Proposition 3.5-5 we have that: $v_{a}(t)=v_{a}(s(u))=$ $s\left(v_{a}(u)\right)=s\left(a \wedge v_{b}(u)\right)=s(a) \wedge s\left(v_{b}(u)\right)=a \wedge v_{b}(s(u))=a \wedge v_{b}(t)$. If $t$ is $u_{1} \wedge u_{2}, v_{a}(t)=v_{a}\left(u_{1} \wedge u_{2}\right)=v_{a}\left(u_{1}\right) \wedge v_{a}\left(u_{2}\right)=\left(a \wedge v_{b}\left(u_{1}\right)\right) \wedge\left(a \wedge v_{b}\left(u_{2}\right)\right)=$ $a \wedge v_{b}\left(u_{1} \wedge u_{2}\right)=a \wedge v_{b}(t)$.

Proposition 5.4 Let $L$ be an $I E_{B}$-lattice and $a, b \in s(L)$ such that $a<b$. Then we have:

$$
\models_{L_{b}} t=r \Longrightarrow \models_{L_{a}} t=r
$$

Proof: By the characterization of equations in $\mathcal{O} \mathcal{M L}$, we study equations of the form $t=1$. Suppose that $L_{b} \models t=1$. Let $v_{a}$ be a $L_{a}$-valuation. By Proposition 5.3 there exists an $L_{b}$-valuation $v_{b}$ such that $v_{a}(\cdot)=a \wedge v_{b}(\cdot)$. Thus $v_{a}(t)=a \wedge v_{b}(t)=a \wedge 1^{L_{b}}=a \wedge b=a=1^{L_{a}}$. Hence $L_{a} \models t=1$.

Proposition 5.4 gives the following useful result: when we consider an arbitrary subvariety $\mathcal{A}_{I}$ of $\mathcal{I E}_{B}$, any interval structure considered in an algebra of $\mathcal{A}_{I}$ lies in $\mathcal{A}_{I}$.

Let $L$ be an orthomodular lattice. It is well known that the map given by $Z(L) \ni z \mapsto \theta_{z}=\left\{(a, b) \in L^{2}: a \wedge z=b \wedge z\right\}$ is a Boolean isomorphism between $Z(L)$ and the Boolean subalgebra of $\operatorname{Con}_{O M L}(L)$ of factor congruences. The correspondence $x / \theta_{z} \mapsto x \wedge z$ defines an $O M L$-isomorphism from 
$L / \theta_{z}$ onto $L_{z}$ and then $x \mapsto(x \wedge z, x \wedge \neg z)$ defines an $O M L$-isomorphism from $L$ onto $L_{z} \times L_{\neg z}$. In what follows we shall establish analogous results for $I E_{B}$-lattices.

Proposition 5.5 Let $\mathcal{A}_{I}$ be a subvariety of $\mathcal{I E}_{B}$. Let $L$ be an algebra in $\mathcal{A}_{I}, z \in s(L)$ and we define the set $\theta_{z}=\left\{(a, b) \in L^{2}: a \wedge z=b \wedge z\right\}$. Then we have:

1. $\theta_{Z} \in \operatorname{Con}_{\mathcal{A}_{I}}(L)$ and $x / \theta_{z} \mapsto x \wedge z$ define a $\mathcal{A}_{\mathcal{I}}$-isomorphism from $L / \theta_{z}$ onto $L_{z}$.

2. $\left(\theta_{z}, \theta_{\neg z}\right)$ is a pair of factor congruences on $L$,

3. The map $s(L) \ni z \mapsto \theta_{z}=\left\{(a, b) \in L^{2}: a \wedge z=b \wedge z\right\}$ is a Boolean isomorphism between $s(L)$ and the Boolean subalgebra of $C_{\mathcal{A}_{I}}(L)$ of factor congruences.

Proof: 1) Let $z \in s(L)$. We first prove that $\theta_{z} \in \operatorname{Con}_{\mathcal{A}_{I}}(L)$. It is well known that $\theta_{z}$ is an $O M L$-congruence. We only need to see the $s$ compatibility. Suppose that $(a, b) \in \theta_{z}$ i.e., $a \wedge z=b \wedge z$. By Proposition 3.5-4 and 5, $s(a) \wedge z=s(a) \wedge s(z)=s(a \wedge s(z))=s(a \wedge z)=s(b \wedge z)=$ $s(b \wedge s(z))=s(b) \wedge s(z)=s(b) \wedge z$. Hence $(s(a), s(b)) \in \theta_{z}$. By Proposition $5.4, L_{z} \in \mathcal{A}$. Let $f: L / \theta_{z} \rightarrow L_{z}$ such that $f\left(x / \theta_{z}\right)=x \wedge z$. Since $f$ is an $O M L$-isomorphism, we have to prove that $f\left(s\left(x / \theta_{z}\right)\right)=s\left(f\left(x / \theta_{z}\right)\right)$. In fact $f\left(s\left(x / \theta_{z}\right)\right)=f\left(s(x) / \theta_{z}\right)=s(x) \wedge z=s(x) \wedge s(z)=s(x \wedge s(z))=s\left(f\left(x / \theta_{z}\right)\right)$. Hence $f$ is a $\mathcal{A}_{I}$-isomorphism.

2) By item 1, $x / \theta_{\neg z} \mapsto x \wedge \neg z$ defines a $\mathcal{A}$-isomorphism from $L / \theta_{\neg z}$ onto $L_{\neg z}$. Thus we have to prove that $g: L \rightarrow L_{z} \times L_{\neg z}$ such that $g(x)=$ $(x \wedge z, x \wedge \neg z)$ is an $\mathcal{A}_{I}$-isomorphism. It is well known that $g$ is an $O M L$ isomorphism, consequently we need to prove that $g(s(x))=s(g(x))$. In fact $g(s(x))=(s(x) \wedge z, s(x) \wedge \neg z)=(s(x) \wedge s(z), s(x) \wedge s(\neg z))=(s(x \wedge z), s(x \wedge$ $\neg z))=s((x \wedge z, x \wedge \neg z))=s(g(x))$. Hence $g$ is an $\mathcal{A}_{I}$-isomorphism and $\left(\theta_{z}, \theta_{\neg z}\right)$ is a pair of factor congruences on $L$.

3) Let $\theta$ be a factor congruence and $h: L \rightarrow L / \theta \times L / \theta^{*}$ be an $\mathcal{A}_{I^{-}}$ isomorphism. Since $h$ is an $O M L$-isomorphism, if we consider the preimage $z=f^{-1}((1,0))$ then, it is well known that $z \in Z(L)$ and $\theta=\left\{(a, b) \in L^{2}\right.$ : $a \wedge z=b \wedge z\}$. Taking into account that $s((1,0))=(1,0)$ we have that: $z=f^{-1}(s((1,0)))=s\left(f^{-1}(1,0)\right)=s(z)$. Hence $z \in s(L)$ and $s(L) \ni z \mapsto$ $\theta_{z}=\left\{(a, b) \in L^{2}: a \wedge z=b \wedge z\right\}$ is a Boolean isomorphism between $s(L)$ and the Boolean subalgebra of $\operatorname{Con}_{\mathcal{A}_{I}}(L)$ of factor congruences. 
If $\mathcal{A}_{I}$ is a subvariety of $\mathcal{I} \mathcal{E}_{B}$ we denote by $\mathcal{D}\left(\mathcal{A}_{I}\right)$ the class of directly indecomposable algebras of $\mathcal{A}$.

Proposition 5.6 Let $\mathcal{A}_{I}$ be a subvariety of $\mathcal{I E}_{B}$. Then we have

1. $L \in \mathcal{D}\left(\mathcal{A}_{I}\right)$ iff $s(L)=\mathbf{2}$.

2. If $L \in \mathcal{D}\left(\mathcal{A}_{I}\right)$ then the function $\sigma_{s}(x)=\left\{\begin{array}{ll}1, & \text { if } s(x)=1^{L} \\ 0, & \text { if } s(x)=0^{L}\end{array}\right.$ is the unique Boolean pre-state coherent with s.

3. Let $L \in \mathcal{D}\left(\mathcal{A}_{I}\right)$ and $x, y \in L$ such that, $x \perp y$ and $s(x \vee y)=s(x) \vee s(y)$. Then $\sigma_{s}(x \vee y)=\sigma_{s}(x)+\sigma_{s}(y)$.

Proof: 1) Follows immediately from Theorem 5.5. 2) Since $s(L)=\mathbf{2}$, by Proposition $4.2,\{1\}$ is the unique prime increasing set in $s(L)$. Hence by Proposition 4.8 and Proposition $4.9, \sigma_{s}$ is the unique Boolean pre-state coherent with $s$. 3) Let $x, y \in L$ such that, $x \perp y$ and $s(x \vee y)=s(x) \vee s(y)$. Suppose that $s(x)=1^{L}$. Then $1^{L}=s(x) \leq s(x \vee y)$ and $s(\neg y)=0^{L}$. Thus $\sigma_{s}(x \vee y)=1, \sigma_{s}(x)=0$ and $\sigma_{s}(y)=1$, i.e., $\sigma_{s}(x \vee y)=\sigma_{s}(x)+\sigma_{s}(y)$. Suppose that $s(x)=0^{L}$. Then $s(x \vee y)=0 \vee s(y)=s(y)$ and $\sigma_{s}(x \vee y)=$ $\sigma_{s}(y)$. Since $\sigma_{s}(x)=0, \sigma_{s}(x \vee y)=0+\sigma_{s}(y)=\sigma_{s}(x)+\sigma_{s}(y)$. Hence $\sigma_{s}(x \vee y)=\sigma_{s}(x)+\sigma_{s}(y)$.

Now we can establish a simple criterium to equationally characterize subclasses of Boolean pre-states.

Theorem 5.7 Let $\mathcal{A}$ be a subcategory of $\mathcal{E}_{B}$ and let $\mathcal{A}_{I}$ be a subvariety of $\mathcal{I E}_{B}$ such that it satisfies the following two conditions:

I: For each $(L, \sigma) \in \mathcal{A}, \mathcal{I}(L) \in \mathcal{D}\left(\mathcal{A}_{I}\right)$ where the internal Boolean prestate in $\mathcal{I}(L)$ is given by $s_{\sigma}(x)= \begin{cases}1^{L}, & \text { if } \sigma(x)=1 \\ 0^{L}, & \text { if } \sigma(x)=0\end{cases}$

E: For each $L \in \mathcal{D}\left(\mathcal{A}_{I}\right),\left(L, \sigma_{s}\right) \in \mathcal{A}$ where $\sigma_{s}$, the unique Boolean prestate coherent with $s$, is given by $\sigma_{s}(x)= \begin{cases}1, & \text { if } s(x)=1^{L} \\ 0, & \text { if } s(x)=0^{L}\end{cases}$

Then $\mathcal{I}: \mathcal{A} \rightarrow \mathcal{D}\left(\mathcal{A}_{I}\right)$ is a categorical equivalence and $\mathcal{A}_{I}$ equationally characterizes $\mathcal{A}$. 
Proof: $\quad$ By condition $\mathbf{E}$ we consider $\mathcal{E}: \mathcal{D}\left(\mathcal{A}_{I}\right) \rightarrow \mathcal{A}$ such that for each $L \in \mathcal{D}\left(\mathcal{A}_{I}\right) \mathcal{E}(L)=\left(L, \sigma_{s}\right)$. If $f: L_{1} \rightarrow L_{2}$ is an $\mathcal{A}_{I}$-homomorphism, by definition of $\sigma_{s_{i}}$ with $i=1,2, \mathcal{E}(f)=f$ is an $\mathcal{A}$-homomorphism. Thus $\mathcal{E}$ is a functor. We prove that the composite functor $\mathcal{E} \mathcal{I}$ is naturally equivalent to the identity functor $1_{\mathcal{A}}$. Let $(L, \sigma) \in \mathcal{A}$. By Proposition 4.10 and Proposition $5.5, \sigma=\sigma_{s_{\sigma}}$. Consequently $\mathcal{E} \mathcal{I}(L, \sigma)=(L, \sigma)$ and $\mathcal{E} \mathcal{I}(f)=f$ for each $\mathcal{A}$-homomorphisms. Then the following diagram is trivially commutative:

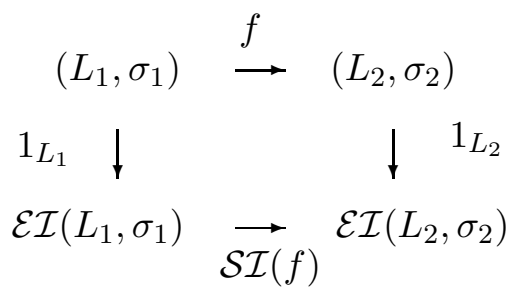

It proves that $\mathcal{E} \mathcal{I}$ is naturally equivalent to the identity functor $1_{\mathcal{A}}$. With analogous arguments we can prove that $\mathcal{I E}$ is naturally equivalent to the

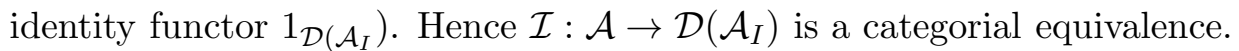

Since $\mathcal{D}\left(\mathcal{A}_{I}\right)$ contain the subdirectly irreducible algebras of $\mathcal{A}_{I}$, it is immediate that $\models_{\mathcal{A}_{I}} t=1$ iff $\models_{\mathcal{D}\left(\mathcal{A}_{I}\right)} t=1$. Hence $\mathcal{A}_{I}$ equationally characterizes $\mathcal{A}$.

Remark 5.8 Let $\mathcal{A}$ be a subcategory of $\mathcal{E}_{B}$. Theorem 5.7 states that every object $(A, \sigma) \in \mathcal{A}$ where $\sigma$ is a two-valued state defined on the orthomodular lattice $A$ is univocally identifiable to a directly indecomposable algebra of the variety $\mathcal{A}_{I}$ and viceversa. In other words, if a class $\mathcal{A}$ of two valued states defined over orthomodular lattices is equationally characterizable through a variety $\mathcal{A}_{I}$ then $\mathcal{A}$ is identifiable to the class of directly indecomposable algebras of $\mathcal{A}_{I}$.

Example 5.9 Boolean pre-states. Let us apply Theorem 5.7 to show that $\mathcal{I E}_{B}$ equationally characterizes $\mathcal{E}_{B}$. I) By Proposition 4.10 , if $(L, \sigma) \in \mathcal{E}_{B}$ then $\mathcal{I}(L) \in \mathcal{I E}_{B}$. E) If $L \in \mathcal{D}\left(\mathcal{I E}_{B}\right)$, by Proposition 5.5, $\left(L, \sigma_{s}\right) \in \mathcal{E}_{B}$. Hence $\mathcal{I E}_{B}$ equationally characterizes the full class $\mathcal{E}_{B}$.

In the next sections we use Theorem 5.7 to characterize two different families of two-valued states. 


\section{Two-valued states}

Now we study the class of two-valued states of Definition 3.1. We denote by $\mathcal{T} \mathcal{E}_{B}$ the full subcategory of $\mathcal{E}_{B}$ whose objects are pairs $(L, \sigma)$ such that $L$ is an orthomodular lattice and $\sigma$ is a two-valued state. We propose the following structure to characterize $\mathcal{T} \mathcal{E}_{B}$.

Definition 6.1 An orthomodular lattice with an internal two-valued state $\left(I T E_{B}\right.$-lattice for short) is a $I E_{B}$-lattice $\langle L, \wedge, \vee, \neg, s, 0,1\rangle$ that satisfies:

$$
s(x \vee(y \wedge \neg x))=s(x) \vee s(y \wedge \neg x)
$$

We denote by $\mathcal{I} \mathcal{T} \mathcal{E}_{B}$ the variety of $I T E_{B}$-lattices.

Proposition 6.2 Let $L$ be a ITE $E_{B}$-lattice and $x, y$ in $L$ such that $x \perp y$. Then $s(x \vee y)=s(x) \vee s(y)$.

Proof: $\quad$ Suppose that $x \leq \neg y$ and then $y \leq \neg x$. Hence, by definition of $I T E_{B}$-lattice, $s(x \vee y)=s(x \vee(y \wedge \neg x))=s(x) \vee s(y \wedge \neg x)=s(x) \vee s(y)$.

Theorem 6.3 $\mathcal{I T}^{\mathcal{T} \mathcal{E}_{B}}$ equationally characterizes $\mathcal{T E}_{B}$.

Proof: We need to prove the two conditions of Theorem 5.7. I) Let $(L, \sigma) \in$ $\mathcal{T} \mathcal{E}_{B}$. We first show that $s_{\sigma}(x \vee(y \wedge \neg x))=s_{\sigma}(x) \vee s_{\sigma}(y \wedge \neg x)$. Since $x \perp y \wedge \neg x, \sigma(x \vee(y \wedge \neg x))=\sigma(x)+\sigma(y \wedge \neg x)$. Then, by Proposition 4.10-2, $s_{\sigma}(x \vee(y \wedge \neg x))=s_{\sigma}(x) \vee s_{\sigma}(y \wedge \neg x)$. Hence by Proposition 5.6-1, $\mathcal{I}(L)=\left(L, s_{\sigma}\right) \in \mathcal{D}\left(\mathcal{I} \mathcal{T} \mathcal{E}_{B}\right)$. $\quad$ E) Let $L \in \mathcal{D}\left(\mathcal{I} \mathcal{W} \mathcal{E}_{B}\right)$ and $x, y \in L$ such that $x \leq \neg y$. By Lemma 6.2, $s(x \vee y)=s(x) \vee s(y)$. Then by Proposition 5.6-3, $\sigma_{s}(x \vee y)=\sigma_{s}(x)+\sigma_{s}(y)$ and $\left(L, \sigma_{s}\right) \in \mathcal{T} \mathcal{E}_{B}$. Hence $\mathcal{I} \mathcal{T} \mathcal{E}_{B}$ equationally characterizes $\mathcal{T} \mathcal{E}_{B}$.

\section{Jauch-Piron two-valued states}

Let $L$ be an orthomodular lattice. A Jauch-Piron two-valued state is a twovalued state $\sigma$ that satisfies

$$
\sigma(x)=\sigma(y)=1 \quad \Longrightarrow \quad \exists c \in L: \sigma(c)=1 \text { and } c \leq x, y
$$

For the analysis of this property imposed by Jauch and Piron [12, 24] we also refer to [31]. We denote by $\mathcal{J} \mathcal{P} \mathcal{E}_{B}$ the full subcategory of $\mathcal{E}_{B}$ whose objects are pairs $(L, \sigma)$ such that $L$ is an orthomodular lattice and $\sigma$ is a Jauch-Piron two-valued state. 
Proposition 7.1 Let $L$ be an orthomodular lattice and $\sigma$ be a two-valued state. Then the following statements are equivalent:

1. $\sigma$ is a Jauch-Piron two-valued state.

2. $\sigma(x)=\sigma(y)=1 \quad \Longrightarrow \quad \sigma(x \wedge y)=1$,

3. $\sigma(x) \cdot \sigma(\neg x \vee y)=\sigma(x \wedge y)$.

Proof: $\quad 1 \rightarrow 2$ ) Suppose that $\sigma(x)=\sigma(y)=1$. By hypothesis there exists $c \leq x, y$ such that $\sigma(c)=1$. Since $c \leq x \wedge y, \sigma(x \wedge y)=1 . \quad 2 \rightarrow 3) \mathrm{We}$ have to consider four possible cases:

Case $\sigma(x)=\sigma(y)=1$. By hypothesis, $\sigma(x \wedge y)=1$. Since $y \leq \neg x \vee y$ we have $1=\sigma(y) \leq \sigma(\neg x \vee y)$. Thus $\sigma(x) \cdot \sigma(\neg x \vee y)=\sigma(x \wedge y)$.

Case $\sigma(x)=1$ and $\sigma(y)=0$. Since $x \wedge y \leq y$ then $\sigma(x \wedge y) \leq \sigma(y)=$ 0 . Note that $1-\sigma(\neg x \vee y)=\sigma(x \wedge \neg y)$. Since $\sigma(x)=\sigma(\neg y)=1$, by hypothesis we have that $\sigma(x \wedge \neg y)=1$ and then $\sigma(\neg x \vee y)=0$. Thus $\sigma(x) \cdot \sigma(\neg x \vee y)=\sigma(x \wedge y)$. The cases with $\sigma(x)=0$ are trivial. Hence $\sigma(x) \cdot \sigma(\neg x \vee y)=\sigma(x \wedge y)$.

$3 \rightarrow 1)$ We first prove that $1-\sigma(x) \cdot \sigma(\neg x \vee y)=\sigma(\neg x) \vee \sigma(x \wedge \neg y)$ where $\vee$ is the supremum in the natural order of $\{0,1\}$. If $\sigma(x)=0$ then $1-\sigma(x) \cdot \sigma(\neg x \vee y)=1$ and $\sigma(\neg x) \vee \sigma(x \wedge \neg y)=1 \vee \sigma(x \wedge \neg y)=1$. If $\sigma(x)=1,1-\sigma(x) \cdot \sigma(\neg x \vee y)=1-\sigma(\neg x \vee y)$ and $\sigma(\neg x) \vee \sigma(x \wedge \neg y)=$ $0 \vee \sigma(x \wedge \neg y)$. Since $1-\sigma(\neg x \vee y)=\sigma(\neg(\neg x \vee y))=\sigma(x \wedge \neg y)$ we have that $1-\sigma(x) \cdot \sigma(\neg x \vee y)=\sigma(\neg x) \vee \sigma(x \wedge \neg y)$.

Suppose that $\sigma(x)=\sigma(y)=1$. Note that $\sigma(\neg x)=0$ and $\sigma(x \wedge \neg y) \leq$ $\sigma(\neg y)=0$. Thus $\sigma(\neg x) \vee \sigma(x \wedge \neg y)=0$ and by the above argument $\sigma(x) \cdot \sigma(\neg x \vee y)=1$. By hypothesis $\sigma(x \wedge y)=1$. Since $x \wedge y \leq x, y, \sigma$ is a Jauch-Piron two-valued state.

Taking into account the last proposition, we propose the following structure to characterize $\mathcal{J P} \mathcal{E}_{B}$.

Definition 7.2 An orthomodular lattice with an internal Jauch-Piron twovalued state $\left(I J P E_{B}\right.$-lattice for short) is a $T E_{B}$-lattice $\langle L, \wedge, \vee, \neg, s, 0,1\rangle$ such that satisfies:

$$
s(x) \wedge s(\neg x \vee y)=s(x \wedge y)
$$

We denote by $\mathcal{I} \mathcal{J P} \mathcal{E}_{B}$ the variety of $I J P E_{B}$-lattices.

Theorem 7.3 $\mathcal{I} \mathcal{J} \mathcal{P} \mathcal{E}_{B}$ equationally characterizes $\mathcal{J} \mathcal{P} \mathcal{E}_{B}$. 
Proof: We need to prove the two condition of Theorem 5.7. I) Let $(L, \sigma) \in$ $\mathcal{J P} \mathcal{E}_{B}$. We first show that $s_{\sigma}(x) \wedge s_{\sigma}(\neg x \vee y)=s_{\sigma}(x \wedge y)$. Suppose that $\sigma(x)=1$. By Proposition 7.1-3, $\sigma(\neg x \vee y)=\sigma(x \wedge y)$ and then $s_{\sigma}(\neg x \vee y)=$ $s_{\sigma}(x \wedge y)$. Thus $s_{\sigma}(x) \wedge s_{\sigma}(\neg x \vee y)=s_{\sigma}(x \wedge y)$. Suppose that $\sigma(x)=0$. By Proposition 7.1-3, $\sigma(x \wedge y)=0$. Thus $s_{\sigma}(x)=0^{L}, s_{\sigma}(x \wedge y)=0^{L}$ and $s_{\sigma}(x) \wedge s_{\sigma}(\neg x \vee y)=0^{L} \wedge s_{\sigma}(\neg x \vee y)=0^{L}=s_{\sigma}(x \wedge y) . \quad \mathcal{I}(L)=$ $\left(L, s_{\sigma}\right) \in \mathcal{D}\left(\mathcal{I} \mathcal{J P} \mathcal{E}_{B}\right)$. $\quad$ E) Let $L \in \mathcal{D}\left(\mathcal{I} \mathcal{J P} \mathcal{E}_{B}\right)$. Let $x, y \in L$ such that $\sigma_{s}(x)=\sigma_{s}(y)=1$. Then $s(x)=s(y)=1^{L}$. Note that $1^{L}=s(y) \leq s(\neg x \vee y)$ and then $1^{L}=s(x) \wedge s(\neg x \vee y)=s(x \wedge y)$. Thus $\sigma_{s}(x \wedge y)=1$. By Proposition 7.1, $\left.\left(L, \sigma_{s}\right) \in \mathcal{J} \mathcal{P} \mathcal{E}_{B}\right)$. Hence $\mathcal{I} \mathcal{J P} \mathcal{E}_{B}$ equationally characterizes $\mathcal{J} \mathcal{P} \mathcal{E}_{B}$.

\section{Conclusions}

In this paper we have developed an algebraic framework in which it is possible to demonstrate that several classes of two-valued states over an orthomodular lattice may be equationally characterized. We have obtained the internalization of a set of classes of two-valued states by enlarging the orthomodular lattice with a unary operator equationally described. This solves the question present in the literature regarding the characterization of several families of two-valued states over orthomodular lattices.

\section{Acknowledgements}

The authors wish to thank an anonymous referee for corrections on an earlier draft of this article, his careful reading and valuable comments.

This work was partially supported by the following grants: PIP 112200801-02543 and Projects of the Fund for Scientific Research Flanders G.0362.03 and G.0452.04.

\section{References}

[1] J. S. Bell, "On the Einstein-Podolsky-Rosen paradox", Physics 1 (1964) 195-200.

[2] G. Birkhoff, and J. von Neumann, "The logic of quantum mechanics", Ann. Math. 27 (1936) 823-843. 
[3] D. Bohm, "A suggested interpretations of the quantum theory in therms of 'hidden variables': Part I', Phys. Rev. 85 (1952) 166-179.

[4] S. Burris, H. P. Sankappanavar, A Course in Universal Algebra, Graduate Text in Mathematics, Vol. 78. Springer-Verlag, New York Heidelberg Berlin, 1981.

[5] M. L. Dalla Chiara , R. Giuntini, R. Greechie, Reasoning in Quantum Theory, Sharp and Unsharp Quantum Logics, Kluwer, DordrechtBoston-London, 2004.

[6] A. Di Nola, A. Dvurečenskij, A. Lettieri, "On varieties of $M V$-algebras with internal states", Int. J. Approx. Reasoning 2009 (to appear).

[7] A. Dvurečenskij, "On States on MV-algebras and their Applications" J. Logic and Computation 2009 (to appear).

[8] A. Einstein, Podolsky and N. Rosen, "Can Quantum-Mechanical Description be Considered Complete?", Phys. Rev. 47 (1935) 777-780.

[9] D. Foulis, "Observables, states, and symmetries in the context of $C B$ effect algebras", Rep. Math. Phys. 60 (2007) 329-346.

[10] R. Godowski, "Varieties of orthomodular lattices with strongly full set of states", Demostratio Mathemamatica XIV 3 (1982) 725-732.

[11] S. Gudder, Stochastic Methods in Quantum Mechanics, Elseiver-NorthHoland, New York 1979.

[12] J. Jauch, Foundations of Quantum Mechanics, Addison Wesley, Reading, Mass, 1968.

[13] J. A. Kalman, "Lattices with involution", Trans. Amer. Math. Soc. 87 (1958), 485-491.

[14] G. Kalmbach, Ortomodular Lattices, Academic Press, London, 1983.

[15] J. Kühr and D. Mundici, "De Finetti theorem and Borel states in [0, 1]valued logic", Int J. Approx. Reason 46 (3) (2007) 605-616.

[16] T. Kroupa, "Every state on semisimple MV-algebra is integral", Fuzzy Sets Syst. 157 (2006) 2771-2787. 
[17] T. Flaminio and F. Montagna, "MV-algebras with internal states and probabilistic fuzzy logics", Int. J. Approx. Reas. 50 (2009) 138152.

[18] J. Harding and P. Pták, "On the set representation of an orthomodular poset", Colloquium Math. 89 (2001) 233-240

[19] F. Maeda and S. Maeda, Theory of Symmetric Lattices, SpringerVerlag, Berlin, 1970.

[20] R. Mayet, "Varieties of orthomodular lattices related to states", Algebra Universalis 20 (1985) 368396.

[21] M. Navara, "Descriptions of states spaces of orthomodular lattices", Math. Bohemica 117 (1992) 305-313.

[22] M. Navara, "Triangular norms and measure of fuzzy set", in Logical, Algebraic, Analytic and Probabilistic Aspect of Triangular Norms, Elsevier, Amsterdan 2005, 345-390.

[23] J. von Neumann, Mathematical Foundations of Quantum Mechanics, Princeton University Press, 12th. edition, Princeton, 1996.

[24] C. Piron, Helv. Phys. Acta 37 (1964) 439.

[25] C. Piron, Foundations of Quantum Physics, Benjamin, Reading, Mass. 1976.

[26] P. Pták, S. Pulmannová, Orthomodular Structures as Quantum Logics, Kluwer, Dordrecht, 1991.

[27] S. Pulmannová, "Sharp and unsharp observables on s-MV algebras A comparison with the Hilbert space approach", Fuzzy Sets Syst. 159, (22) 2008 3065-3077.

[28] P. Pták, "Weak dispersion-free states and hidden variables hypothesis", J. Math. Phys. 24 (1983) 839-840.

[29] Z. Riečanová, "Continuous lattice effect algebras admitting ordercontinuous states", Fuzzy Sets Syst. 136 (2003) 41-54.

[30] Z. Riečanová, "Effect algebraic extensions of generalized effect algebras and two-valued states", Fuzzy Sets Syst. 159 (2008) 1116-1122. 
[31] G.T. Rüttimann "Jauch-Piron states", J. Math. Phys. 18 (1977) 189193.

[32] J. Tkadlec, "Partially additive measures and set representation of orthoposets", J. Pure Appl. Algebra 86 (1993) 79-94.

[33] J. Tkadlec, "Partially additive states on orthomodular posets", Colloquium Mathematicum LXII (1995) 7-14.

[34] J. Tkadlec, "Boolean orthoposets and two-valued states on them", Rep. Math. Phys. 31 (1992) 311316. 